\title{
Der Kalte Krieg und das Meer
}

\author{
Zur Erinnerungspolitik des U-Boot-Films
}

Johannes Pause

\begin{abstract}
Military exploits in the Cold War often differed from conventional acts of war particularly in that they were, by necessity, invisible: In order to prevent nuclear conflict, crises needed to be kept secret, and were therefore not inscribed into cultural memory. Nuclear submarines are paradigmatic of this dynamic: As mobile nuclear missile silos, they played a central role in the balance of terror during the Cold War, effective because they remained passive and invisible. Cold War era submarine films, however, followed old genre traditions by conceiving the submarine as a tactical, mostly explorative and active weapon. It was not until the 199 os that a reboot of the genre emerged, which sought to make the depths of the ocean visible and memorable as a strategic location of the Cold War. Rather than portraying pure military actions, these movies tell stories of intercultural negotiation which, to prevent the outbreak of nuclear war, had to remain hidden under the surface of the sea.
\end{abstract}

Title: The Cold War and the Sea: Politics of Memory and the Submarine Film

Keywords: submarine; Cold War; Hollywood; film studies; memory

\section{Ein Krieg ohne Monumente}

In The Hunt for Red October (USA 1990), John McTiernans einflussreicher Verfilmung des gleichnamigen Romans von Tom Clancy (vgl. 1984), fasst der russische U-Boot-Kapitän Ramius (Sean Connery) das erinnerungspolitische Dilemma des Kalten Kriegs zusammen: »It's a war with no monuments, only casualties.« Der Konflikt der Großmächte findet wesentlich im Geheimen statt, in Sphären der Klandestinität, und seine Opfer bleiben ebenso unbekannt wie seine (zumeist männlichen) Helden, die in der Regel nicht Soldaten, sondern Spione, Analysten, Think-Tank-Mitarbeiter, Wissenschaftler und Zivilisten sind - oder aber, wie Ramius andeutet: die Kapitäne sowjetischer wie US-amerikanischer Atom-U-Boote. Die strategische Bedeutung dieses Schiffstyps war für die Logik der nuklearen Abschreckung von entscheidender Bedeutung, handelte es sich doch um die ultimative Zweitschlagwaffe: Auch wenn ein Erstschlag sämtliche Raketensilos auf dem Boden des eigenen Territoriums treffen und auslöschen würde, könnte jedes der mobilen, in den Tiefen des Meeres nicht ortbaren, aber mit mehreren Atomraketen bestückten U-Boote allein einen Gegenschlag durchführen und so zumindest die urbanen Zentren der feindlichen Macht der Vernichtung preisgeben. Der Versuch, die Atom-U-Boote des Gegners 
durch - ebenfalls mit Atomkraft betriebene - Jagd-U-Boote beständig verfolgen und ausspionieren zu lassen, bildet daher eines der klassischen Katz-und-MausSpiele des Kalten Kriegs, das McTiernans Film akkurat wiedergibt (vgl. Koldau 2010: 269) und mit dem neuartigen Raupenantrieb des titelgebenden Schiffs namens Roter Oktober, der für das amerikanische Sonar fast - natürlich nur fast unhörbar ist, auf die Spitze treibt.

Im Gegensatz zu den U-Booten des Zweiten Weltkriegs sind die zunehmend atomar betriebenen U-Boote des Kalten Kriegs in erster Linie keine taktischen, sondern strategische Waffen (vgl. Koldau 2012: 112). Selbst wenn sie Spionageaufgaben wahrnehmen, ist ihr Wert nicht von spontanen Aktionen ihrer Kapitäne abhängig, sondern basiert vor allem auf ihrer kontinuierlichen, aber unbemerkten Anwesenheit. So tauchen sie auch nicht nur in jenen Situationen, in denen sie angreifen oder verfolgt werden, sondern bewegen sich monatelang unter Wasser auf festen Routen, die sie durch mehrere Ozeane führen können. Nuklear betriebene U-Boote wurden mithin regelrecht dazu konzipiert, das Hoheitsgebiet eines Staates in den nomadischen Raum des Ozeans zu verschieben, in dem sie sich als mobile Exklaven fernab anderer Hoheitsgebiete bewegen können. Ein Atom-U-Boot bringt dabei nahezu alles mit, was einen Staat ausmacht, und immer wieder werden in Spielfilmen und Serien Szenarien entwickelt, in denen die Besatzung eines solchen Schiffes sich für unabhängig erklärt und das Boot selbst als dritte Macht in die Balance des Kalten Kriegs eingreifen lässt - so etwa in dem James-Bond-Film The Spy Who Loved Me (USA 1977, R.: Lewis Gilbert), in Buried on Sunday (CN 1992, R.: Paul Donovan) oder in der Serie Last Resort (USA 2012-13, R.: Karl Gajdusek, Shawn Ryan), die dieses Szenario in die Gegenwart verlegt. Häufig erscheinen U-Boote auch als letztes Refugium der Menschheit nach einem nuklearen Weltkrieg, wie etwa in On the Beach (USA 1959, R.: Stanley Kramer). Tony Scott hebt in Crimson Tide (USA 1995) die globalpolitische Bedeutung der Waffe in einem dem Film vorangestellten Vergleich deutlich hervor:

The three most powerful men in the world:

The President of the United States...

The President of the Russian Republic...

and...

The Captain of a US Nuclear Missile Submarine

(Crimson Tide)

Als Quasistaat ist das Atom-U-Boot jedoch auch deshalb von Bedeutung, weil es seit dem Ende des Kalten Kriegs eine relativ einheitliche fiktionale Ausgestaltung erfahren hat, in der die Grenzen zwischen den großen machtpolitischen Blöcken und mithin auch jene zwischen >westlicher< und >östlicher< Kultur zu einem gewissen Grad verschwimmen. Russische und amerikanische Kommandanten geben in den Fiktionen ähnliche Befehle, sehen sich mit vergleichbaren Problemen konfrontiert und finden sich in Krisensituationen gleichermaßen in der Situation wieder, sich gegen die globalstrategische Vorgehensweise ihres ei- 
genen Landes stellen zu müssen. Eben in jener Zeit, in der Erinnerung zu einer zentralen diskursiven Währung im Hollywoodkino wird (vgl. Grainge 2003: 4), bildet das Genre des U-Boot-Films, so soll im Folgenden argumentiert werden, auf diese Weise einen fiktionalen Handlungsraum aus, der als transnationaler Erinnerungsraum funktionalisierbar wird. Dieser Handlungsraum ist vor allem durch die von Ramius beschriebene Sichtbarkeitsproblematik charakterisiert: Als - für das Hollywoodkino typische - allegorische Figur der Staatlichkeit (vgl. Gradinari 2018) sieht sich der U-Boot-Kapitän mit einer regelrechten Verhinderung von Erinnerbarkeit beauftragt, die nicht nur durch die partielle Klandestinität der Waffe, sondern auch durch ihre Passivität begründet ist. Würde das UBoot als Agent auf den Plan treten, würde das die Mechanik des Atomkriegs in Gang setzen und potenziell mit der Menschheit auch die Möglichkeit jeder Erinnerung auslöschen; solange es hingegen unsichtbar bleibt, ist der Frieden weiterhin gesichert. Auch Heldentaten können daher letztlich nur in der Verweigerung hervorstechender und erinnerbarer Kriegshandlung bestehen.

Für die filmischen Erzählungen, die sich um eine erinnerungskulturelle Aufbereitung des Kalten Kriegs und vor allem der zentralen Bedeutung des AtomU-Boots bemühen, stellt dies eine besondere Herausforderung dar: Um im Rahmen eines Narrativs erinnerbar zu werden, muss die Passivität des Nichthandelns in die Aktivität einer Entscheidung oder Handlung übersetzt werden, was in der Regel durch eine systemische Abdichtung des geschlossenen Handlungsraums U-Boot möglich wird. Zu dieser Abdichtung erweist sich das UBoot deshalb als besonders geeignet, weil es - darin vergleichbar dem Atombunker - einen hermetisch abgeschlossenen »Experimentalraum « (Nanz 2016: 40) ausbildet, an dem sich zentrale Probleme der politischen Epistemologie - wie können wir wissen, was der Feind im Sinn hat? - sowie der Souveränität und Handlungsmacht - welcher Akteur trifft letztlich die Entscheidung über die Zukunft der Welt? - durchspielen lassen. Im Binnenraum des zur »Heterotopie« (Foucault 2005) und damit auch zur »Szenographie« (Eco 1987: 98) geronnenen Schiffs werden im Fall des erinnerungspolitischen U-Boot-Films dabei privilegiert solche Handlungsfolgen erzählt, die letztlich zum Resultat haben, dass auf der Bühne der internationalen Politik das Ereignis des Kriegs ausbleibt: Analysen und Handlungen der Besatzung des Bootes führen dazu, dass dieses unsichtbar bleibt.

Vor diesem Hintergrund wird verständlich, dass viele der Helden, von denen die US-amerikanischen Erinnerungsfilme handeln, Sowjets sind. Nach dem Ende der tatsächlichen Konfrontation entwickelt Hollywood eine filmische Erinnerungskultur, die nicht nur als aktuelles politisches Instrument der Verständigung mit dem ehemaligen Feind dienen soll (vgl. Rayner 2007: 192), sondern den Aufschub der eigenen Vernichtung in die Form eines heroischen Narrativs zu transformieren sucht, das sinnvoll nur einem feindlichen Akteur zugeschrieben werden kann, der sich der Zwangsläufigkeit des Kriegs widersetzt. Anders gesagt: Die »Interkulturalität des Krieges« (Mann 2018) stellt sich im Fall des durch kulturelle Separation und eine unvollständige, phantasmagorische Imagination des Feindes (vgl. Robin 2009) gekennzeichneten Konfliktes der Super- 
mächte allein in der fiktionalen Nachbereitung ein. Bezug genommen wird in den Filmen dabei auf historische Krisen, also jene Fast-Ereignisse, die sich durch die gesamte Geschichte des Kalten Kriegs hindurch verfolgen lassen, deren tatsächliche Hintergründe von beiden Supermächten jedoch in der Regel unter Geheimhaltung gestellt wurden. In diese Lücke stößt der US-amerikanische Spielfilm, nicht indem er die historische Wirklichkeit investigativ erforscht, sondern indem er sie mit Mythen füllt. Retrospektiv werden auf diese Weise die Monumente dieses nicht stattgefundenen Kriegs hergestellt, wobei auf The Hunt for Red October weitere »Erinnerungsfilme« (Erll/Wodianka 2008: 1) wie Crimson Tide, der den Kalten Krieg bereits in die asymmetrische Situation einer terroristischen Bedrohung übergehen lässt (vgl. Koldau 2010: 302), Kathryn Bigelows K-19: The Widowmaker (USA 2002) oder Todd Robinsons Phantom (USA 2013) folgen. Sie beziehen sich teilweise auf historische Fälle, deren Details bis heute nicht vollkommen aufgeklärt sind. Die meisten dieser Werke sind von erinnerungspolitischem Pathos geprägt: Die Helden der submarinen Abschreckung, die ihr Leben gaben, um den Atomkrieg zu verhindern, werden hier tatsächlich in Zeremonien geehrt (Abb. 1). Grundlage dieser Würdigung ist jedoch stets das memorialpolitische immutable mobile (vgl. Latour 1986) ${ }^{1}$ des U-Boots, dessen Ausgestaltung zugleich eigentümliche interkulturelle Spiegelungen und Verschränkungen zwischen West und Ost bedingt.

Abbildung 1: In Kathryn Bigelows K-19: The Widowmaker gedenken die Überlebenden der Katastrophe von 1961 im Jahre 1989 ihren verstorbenen Kameraden.

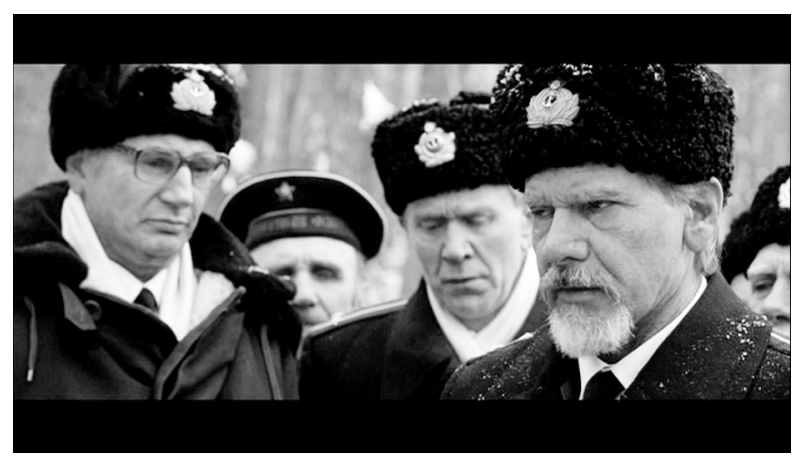

1 Der Begriff bezeichnet bei Latour ursprünglich vor allem wissenschaftliche Objekte wie etwa Karten oder Bücher, die transportabel sind, dabei in Form und Funktion jedoch konstant bleiben. 


\section{Chiffren der Handlungsmacht: das Atom-U-Boot im Kalten Krieg}

Das erinnerungspolitische »kleine Genre« (Brinckmann/Lowry/Wulff 2014) der 1990er Jahre unterscheidet sich erheblich von jenen Werken, die sich in der Zeit des Kalten Kriegs selbst dem Atom-U-Boot widmen. Diese Filme können sich lange Zeit nicht von den Narrativen lösen, die mit dem U-Boot der pränuklearen Ära verbunden sind: Es bleibt hier ein weiterhin taktisch eingesetztes Vehikel tollkühner Abenteurer und Draufgänger, das gerade nicht als Bestandteil einer militärischen Gesamtstrategie der mutual assured destruction - des Gleichgewichts des Schreckens - auf den Plan tritt, sondern für besondere Aufgaben und geheime Missionen eingesetzt wird (vgl. Koldau 2012: 115). Etwa in Samuel Fullers Hell and High Water (USA 1954) oder in John Sturges Ice Station Zebra (USA 1968) besteht diese Mission darin, an unzugänglichen Orten der Welt - in fremden Hoheitsgewässern sowie am Nordpol - verdächtige Vorgänge aufzuklären, wobei die Filme in der Regel gemeinsam mit den Booten in die Räume des Geheimen vordringen. Das U-Boot dient hier als exploratives Medium, das Grenzbereiche des Wissens ebenso erkundet wie einen zwar nur unter Gefahren, aber doch grundsätzlich präzise ortbaren, zudem oftmals durch rassische Klischeebilder als >anders merksamkeit, die in diesen Filmen auf das mediale Instrumentarium und die teleskopische Epistemologie der Boote gelegt wird (Abb. 2), macht diese implizit zum Komplizen der Kamera: U-Boot und Kino folgen gemeinsam dem Auftrag, die Arkanbereiche des Kalten Kriegs sicht- und hörbar werden zu lassen.

Hell and High Water etwa beginnt mit einer Explosion, die, wie sich erst im Verlauf der Handlung herausstellen wird, von der Mannschaft eines amerikanischen U-Boots ausgelöst wurde. Der Film folgt der Mission der Besatzung und klärt somit die Vorgeschichte des Ereignisses auf, mit dem die Machenschaften der Großmächte im Kalten Krieg plötzlich sichtbar wurden. Durch die Retrospektion erhält das Ereignis Sinn, wird in ihr doch gezeigt, wie die Mannschaft die Vorbereitungen Nordkoreas für einen Atomschlag entdeckt und diesen verhindert. Der unsichtbare Raum unter dem Meeresspiegel wird auf diese Weise mit dem klandestinen Raum des Kalten Kriegs kurzgeschlossen; das Ziel der Tauchfahrt besteht in einem »Sichtbar-Machen der eigentlich unsichtbaren Konflikte« (Grob 2010: 228). Die Filme weisen dabei kuriose Verdrehungen der tatsächlichen strategischen Bedeutung der Waffe auf: In Ice Station Zebra geht es etwa um Satellitenbilder, auf denen alle Atomraketen in den USA und der UdSSR zu sehen sind und die nach dem Absturz des Satelliten von der Mannschaft des Bootes gefunden werden müssen. Dass Atom-U-Boote in Wirklichkeit selbst als nichtstationäre Raketensilos fungieren, bleibt unerwähnt; die skeptische Frage, ob das eigene U-Boot mit Atomraketen bestückt sei, wird vom Kapitän vehement verneint. Die geheime Mission, auf die sich die Mannschaft begibt, stellt auf diese Weise eine eigentümliche Verdrehung, wenn nicht gar eine Verschleierung der eigentlichen Zwecke der Waffe dar. 
Abbildung 2: In John Sturges Ice Station Zebra dient das mediale Instrumentarium des U-Boots der Erforschung der Außenwelt.
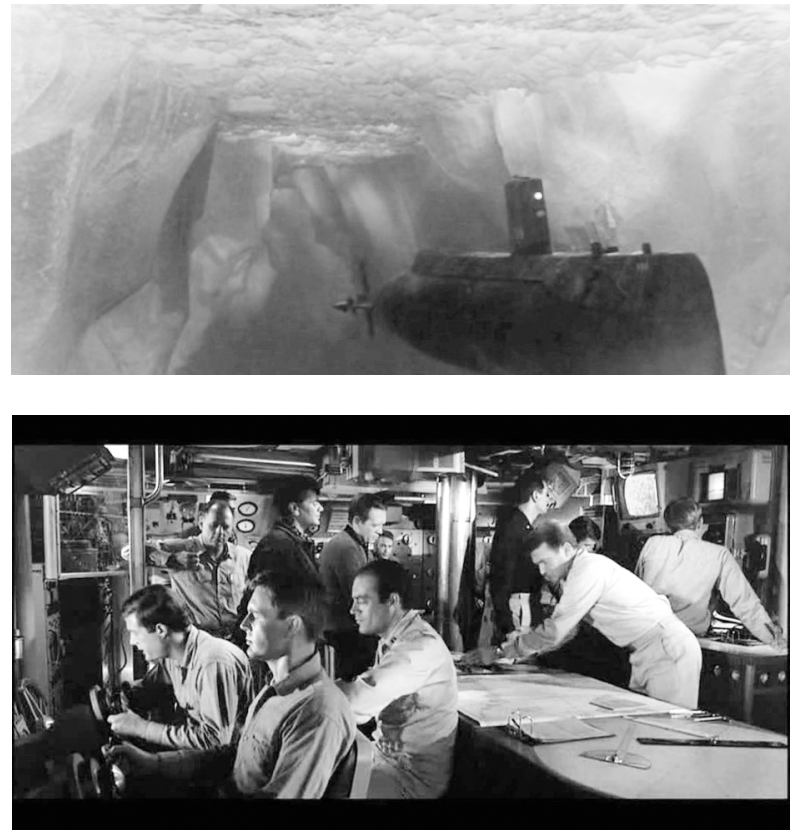

Die starken Subjektivierungsgesten, die den U-Boot-Film kennzeichnen, erscheinen dabei als Kompensation der durch die Individuen insgesamt nicht mehr beeinflussbaren »Globalstrategie« (Engell 1989) des Kalten Kriegs. U-Boot-Kapitäne agieren im Rahmen des Abenteurernarrativs ähnlich wie Ritter, die sich - von Eisen ganz umhüllt und daher nur bedingt bewegungsfähig, dafür jedoch mit lanzenartigen Torpedos bewaffnet - als souveräne Agenten des Schlachtfelds miteinander duellieren. Der Zweikampf ist der Modus ihrer Konfrontation, und in ihrer Ritterlichkeit sind die Vertreter der beiden Weltmächte im Prinzip vollkommen identisch. Sie verhalten sich nicht nur spiegelbildlich, jedes Duell ist auch eine Kommunikation, in welcher die Finten und Tricks des Gegners antizipiert, verstanden, bewundert und gekontert werden müssen. Dabei steht in der Regel der Ausgang des Kriegs insgesamt auf dem Spiel; der tapfere und kluge Kampf der Kapitäne ist zugleich von weltpolitischer Bedeutung. Ein solches Duell kann sich lange hinziehen, da es immer wieder vorkommt, dass die Kapitäne etwa die Schiffe auf Grund laufen lassen, um sich dem Sonar des Gegners zu entziehen. Die visuelle Ähnlichkeit der sich duellierenden Boote, die erneut ihr begrenztes Sensorium kenntlich macht, spiegelt dabei die binäre Logik des Kalten Kriegs, die auf einer Imagination des Anderen basiert, welcher niemals allein als absoluter Feind, sondern immer auch als Maßstab und Spiegel des Selbst fungiert (Abb. 3). 
Abbildung 3: In Samuel Fullers Hell and High Water verhalten sich die U-Boote und ihre Besatzungen spiegelbildlich zueinander.
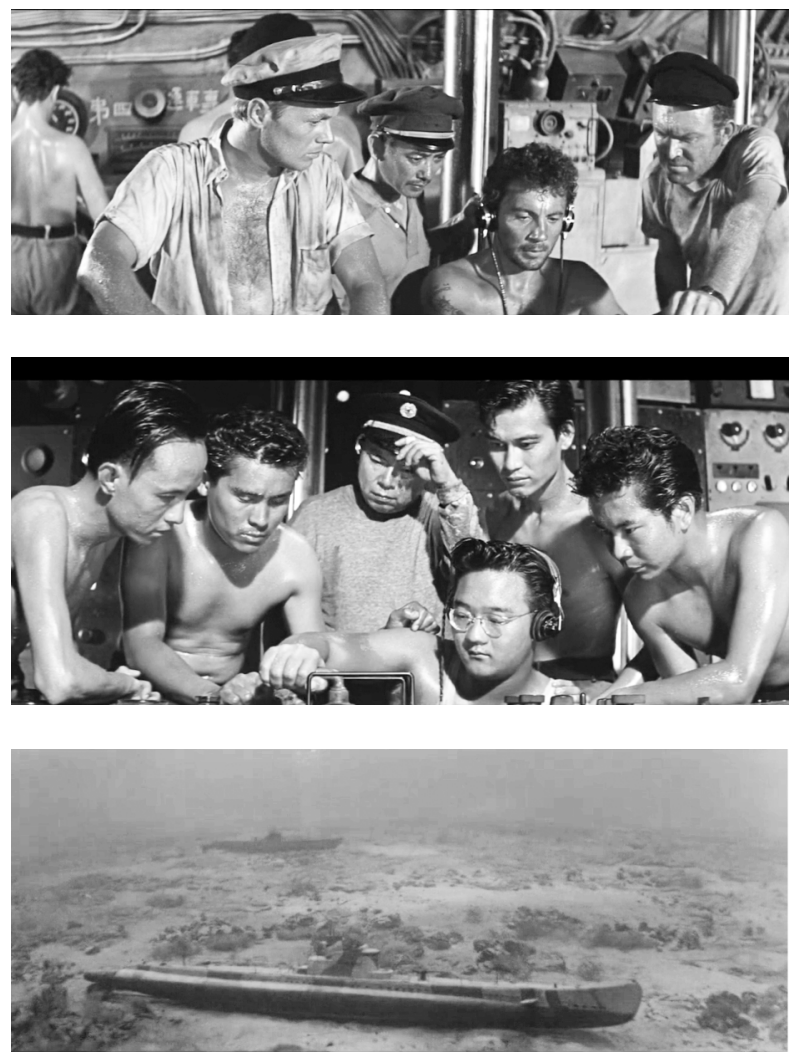

Der U-Boot-Film der $1950 e r$ und $1960 e r$ Jahre verbindet mithin die Logik des Kalten Kriegs mit der Faszination des Entdeckungs- und Abenteuerfilms. Dabei wird die Unterwasserwelt immer wieder zu einem phantasmagorisch aufgeladenen Raum, der unheimliche Wesen und phantastische Formationen hervorbringt. Innen- und Außenraum stehen sich als Räume der Technik und der Natur gegenüber und fallen auch filmästhetisch sichtbar auseinander. Der Raum des Feindes wird zugleich dem Außenraum zugeschrieben; die Reise bewegt sich in ein mehrfach als gefahrvoll markiertes, da prinzipiell feindliches Gewässer. Die Grenze zwischen Kalter-Krieg-Reflexion und Abenteuernarrativ verschiebt sich dabei im Verlauf der Handlung und lässt immer neue Genreübergänge entstehen, etwa zum Katastrophenfilm oder zum phantastischen Film. Voyage to the Bottom of the Sea (USA 1961, R.: Irwin Allen) zum Beispiel spielt auf Jules Verne an, handelt aber zugleich von einer durch Meteoriten hervorgerufenen Klimaerwärmung, gegen die das U-Boot vorgehen muss, und verbindet auf diese Weise Wissenschaftsfiktion, Phantastik, Katastrophenszenario und - zumindest metaphorisch und darin den Invasionsfiktionen dieser Zeit (vgl. Seed 
1999: 132f.) vergleichbar - die Angst vor einem vernichtenden Angriff auf das eigene Territorium. Es finden sich hier mithin Imaginationen eines möglichen Untergangs, in denen sich die reale Gefahr der nuklearen Auslöschung zwar abschattet, die diese jedoch in deutlich als fiktional zu erkennende, mit handlungsmächtigen Subjekten versehene Szenarien verschieben. Die Filme erweisen sich auf diese Weise zugleich als epistemisch und eskapistisch, werden in ihnen doch die Funktionsweisen des Kalten Kriegs teilweise metaphorisch verdeutlicht, teilweise imaginativ entrückt.

\section{Die Szenographie als Erinnerungsort}

Im Gegensatz zu diesen Fiktionen aus den Zeiten des Kalten Kriegs steht die Renaissance des Genres im Modus des Erinnerungsfilms um 1990 für eine realistische Wende: Auch wenn es sich etwa bei dem Raupenantrieb der Roten Oktober um eine Fortschreibung der paranoiden Imaginationen des Feindes im Kalten Krieg handelt, ${ }^{2}$ wird nun im Wesentlichen mit realen U-Booten gedreht, nicht mehr mit Modellen, und die strategische Bedeutung der Waffe wird in den Fiktionen deutlich kenntlich gemacht. Besondere Missionen sind nun als Ausbruch aus der globalstrategischen Rolle der Waffe gekennzeichnet und dienen daher nicht länger der Aufklärung, sondern der militärischen Eskalation - oder, wie in The Hunt for Red October, auf Umwegen letztlich deren Prävention. Zugleich orientiert sich die Darstellung der Boote anders als in den Vorgängern nicht mehr an der Metapher des schnellen und kämpferischen Hais, sondern an jener des global agierenden, schwerfälligen und mächtigen Wals (vgl. Koldau 2012: 110).

Das Imaginäre wird in den neueren Filmen dabei zum Bestandteil der Narrative selbst: Ins Zentrum der Kommunikation zwischen Schiff und Außenwelt rücken nun Versuche, die Absichten des Feindes zu erraten, sowie die Berechnung von Wahrscheinlichkeiten. Sollten die Amerikaner nicht verstehen, dass die Roter Oktober überlaufen will, und stattdessen von der Annahme ausgehen, dass es sich bei ihrem Manöver um einen Angriff handelt, werden sie möglicherweise selbst zum Angriff übergehen - eine Option, die sowohl Ramius als auch die sowjetischen Politiker wiederum einkalkulieren und zu verhindern versuchen, indem sie durch bestimmte Hinweise und cues die Wahrnehmung des Gegenübers steuern. Die Decodierung medial erzeugter Bilder referiert dabei einerseits auf die Kinoerfahrung der Zuschauenden, die nach David Bordwell ebenfalls durch cues gesteuert wird (vgl. Bordwell 1992), und hebt so die politische Bedeutung technischer Medien hervor, die im Kalten Krieg immer schon in den Entscheidungsprozess eingebunden sind (vgl. Nanz 2016). Andererseits besteht in eben dieser anhaltenden Abfolge aufeinander reagierender Imaginationen und Berechnungen das eigentliche Charakteristikum des Kalten Kriegs,

2 | "The endowment of Soviet submarines with arcane abilities in the novel and film derives from highly speculative late-Cold War analyses, used to justify the American naval build-up supervised by Reagan and Lehman." (Rayner 2007: 198) 
den Eva Horn als ein »Gedankenexperiment « beschrieben hat, »dessen logisches Ergebnis immer wieder, in immer neuen Konfliktberechnungen, darin besteht, das Experiment nie in Wirklichkeit umzusetzen.« (Horn 2007: 222) In diesem Experiment geht es um Handlungsoptionen und -verkettungen und daher um mögliche Zukünfte, und so thematisiert auch der Erinnerungsfilm letztlich vergangene Zukünfte und alternative Geschichtsverläufe, die haarscharf nicht eingetreten sind, und jene Helden, die diese Verläufe in einem besonderen Moment der Wahl durch die Akzeptanz des Risikos, die Lage falsch einzuschätzen, aktiv verhindert haben.

Die Tiefsee erweist sich für dieses spieltheoretisch grundierte Geschehen als idealer Schauplatz, denn er

zeichnet sich durch eine vielfache Verschränkung von Medien und Technologien der Sichtbarmachung und des Unsichtbarwerdens aus [...]: Es ist ein Raum, der von Strategien und Taktiken des Versteckens und Entdeckens strukturiert wird; und nicht zuletzt ein Raum, dessen mediale Potenziale selbst technisch genutzt und operationalisiert werden. (Vehlken 2018: 358)

Im Fokus der Handlung des U-Boot-Films steht daher immer auch ein Konflikt zwischen unterschiedlichen Interpretationsrastern, wobei die Wahl des Rasters expliziert werden muss, da sie über den Fortbestand der Menschheit entscheiden kann - das Genre wird von einem »Pathos des schlimmsten Falls« (Horn 2009: 95) regelrecht regiert. Das U-Boot ist mithin kein quasifilmisches Medium der Aufklärung einer letztlich unzweifelhaften Welt mehr, sondern Ort ebenso wie Gegenstand einer globalen intelligence, die Wirklichkeit immer schon als problematisch begreift. Wie der Raupenantrieb der Roten Oktober kenntlich macht, kann der Raum der Tiefsee immer nur begrenzt gekerbt werden; den Techniken der Sichtung und Ortung stehen stets Verfahren einer »erneuten Raumglättung «(Vehlken 2018: 362 ) gegenüber. Aus diesem Grund geht es auch nicht länger um den Helden als Abenteurer, sondern um den Strategen, den Querdenker, den Abhörspezialisten oder gar den Spieler als Helden (vgl. Horn 2007) - um Figuren also, die mit feinsten Spuren und Indizien, aber auch mit den Annahmen und Glaubenssätzen der anderen immer schon kalkulieren.

Diese interpretative Dimension wird in einer Szene von The Hunt for Red October besonders deutlich, in der es Ramius unter Vorspiegelung falscher Tatsachen gelungen ist, den Großteil seiner russlandtreuen Mannschaft zu evakuieren. Die Matrosen wissen nichts von seinen Plänen, zu den Amerikanern überzulaufen, und so denken sie, dass er sich mit amerikanischen Schiffen ein wildes Gefecht liefert, während er tatsächlich mit Hilfe der inzwischen an Bord gekommenen amerikanischen Offiziere gegen ein russisches Jagd-U-Boot kämpft. Den ganzen Film hindurch sind die Manöver der Roten Oktober Gegenstand der Interpretation verschiedener Personen. Richtig verstehen tut sie nur der junge CIA-Analyst Jack Ryan (Alec Baldwin), der in der Lage ist, sich in Ramius hineinzuversetzen und - unter Akzeptanz der Prämisse, dass der feindliche Kommandant tatsächlich überlaufen möchte - zu antizipieren, was dieser 
tun wird. Imagination und Einfühlung werden so - als Tätigkeiten, die ohne den festen Boden verlässlichen Wissens operieren - als überlebenswichtige politische Arbeit etabliert; das U-Boot wird aus erinnerungstheoretischer Perspektive zugleich zu einem Ort der »Heterochronie« (Große Kracht 2014: 70), an dem vergangene Zukunftserwartungen nacherlebt werden können. Auffällig ist dabei, dass die tatsächlichen Reflexe des Kalten Kriegs - hinter allen Ereignissen die Arbeit des Feindes am Werk zu vermuten - gerade überwunden werden müssen: Der im Jahr 1990 entstandene Film stellt mithin den schwierigen Prozess des Einübens neuer Verhaltensweisen, die gerade nicht denjenigen des Kalten Kriegs entsprechen, selbst zur Schau (vgl. Sharp 1998: 162).

War das Verhältnis zwischen Innenwelt des U-Boots und seiner Umgebung in den früheren Filmen noch unproblematisch, während beide Welten filmästhetisch tendenziell auseinanderfielen, verhält es sich nun andersherum: Während die Zuschauenden den Zusammenhang zwischen den über den ganzen Globus verteilten, parallel montierten Schauplätzen der Handlung - etwa Weißes Haus, Kreml, Nato-Stützpunkte und andere Schiffe - in Gänze überblicken, wird die Kommunikation zwischen U-Boot und Außenwelt auf inhaltlicher Ebene problematisch. Gerade durch diesen Gegensatz wird der Raum des U-Boots als heterotoper etabliert: Je länger es unter Wasser bleibt, desto stärker gehorcht es seiner hermetischen Eigenlogik (zum heterotopen Charakter submariner Welten vgl. Ritzer 2010). In der Außenwelt wird etwa zum Thema, wie Massenmedien die Vorgänge um das Boot interpretieren - in Crimson Tide zum Beispiel durch die Integration des in den 1990 or Jahren populären Nachrichtensprechers Richard Valeriani, der von den Geschehnissen berichtet. Der Innenraum des Bootes wird zugleich dadurch regelrecht als abgeschlossener Organismus etabliert, dass er in allen Details erklärt wird. Wie schon in den U-Boot-Filmen der 1950er Jahre - so zeigt Irina Gradinari in ihrem Beitrag zu diesem Heft - wird oftmals zu Beginn des Films ein Neuling in die Funktionsweise der verschiedenen Stationen eingeführt, deren räumlicher Zusammenhang sich infolge seiner Initiation auch für die Zuschauenden herstellt. Diese Stationen stehen als schiffsinterne Öffentlichkeit den beschränkten Privaträumen der Mannschaft gegenüber, sodass das Boot insgesamt als Miniatur der Gesellschaft erscheint. Aus erinnerungspolitischer Sicht dient die Spannung, die die Filme zwischen Außen und Innen herstellen, der Erläuterung des Vergessens: Es wird gleichzeitig geschildert, was tatsächlich geschah und weshalb es nicht erinnert wurde, was die Mannschaft unter Wasser durchmachte und wie sich die Außenwelt dazu verhielt - eine Perspektivierung des Geschehens, die den früheren, explorativen Werken des Genres weitgehend fehlt. Geschichte wird als diskursives Konstrukt bewusst, das von den Filmen einer Revision unterzogen wird, indem sie Verborgenes sichtbar machen; doch ist dieses Verborgene nun nicht mehr ein Geheimnis an einem verborgenen Punkt auf dem Globus, das durch das Medium des U-Boots ausspioniert wird, sondern das Geschehen an Bord selbst.

Folgerichtig konzentrieren sich die Filme nun auch weitaus stärker auf die klaustrophobische Atmosphäre im Inneren des Bootes, als dies in früheren Werken der Fall war. Auch hierfür ist der Wechsel zwischen Innen- und Außenper- 
spektiven entscheidend: Der Film Crimson Tide etwa reproduziert die alten Kalter-Krieg-Narrative aus Filmen wie Fail Safe (USA 1964, R.: Sidney Lumet), in denen ein nichtbestätigter Befehl vorliegt, den Atomkrieg auszulösen, und es kein Mittel zur Entscheidung gibt, ob dieser Befehl gültig ist oder nicht. Der Kontakt zur Außenwelt ist gekappt, die Offiziere des Bootes müssen die strategische Entscheidung allein treffen. Im Angesicht dieser Aufgabe reproduziert sich das Motiv des Duells im Innenraum des Schiffes. So versuchen in den Werken immer wieder Saboteure oder Geheimdienstmitarbeiter, das Kommando an sich zu reißen, und oftmals geht das Boot rogue, verhält sich also entgegen seinen eigentlichen strategischen Einsatzzielen. Der Konflikt zwischen den verschiedenen Fraktionen an Bord wird dabei in aller Regel durch zwei Autoritätsfiguren ausgetragen (vgl. Koldau 2010: 309; Grob 2010: 235f.), die jeweils einen Teil der Mannschaft hinter sich bringen oder diktatorisch dominieren (»We are here to preserve democracy, not to practice it«, heißt es in Crimson Tide einmal) und die stellvertretend für die politischen Antagonismen stehen, die auch außerhalb des Bootes den politischen wie militärischen Diskurs grundieren (Abb. 4).

Abbildung 4: In Crimson Tide reproduziert sich das Duell im Innenraum des Bootes, während die Außenwelt nun Gegenstand einer ausufernden Interpretation geworden ist.

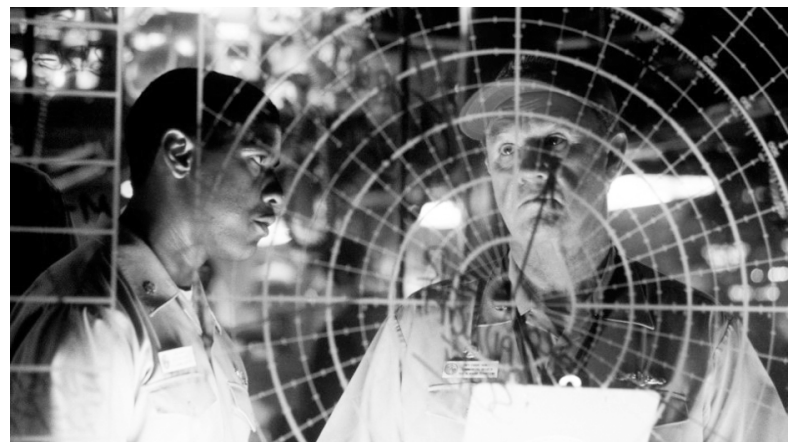

Innerhalb des Bootes reproduzieren sich so die äußeren Fronten - teilweise inklusive der gesellschaftlichen Spaltungen, die sich etwa anhand ethnischer Differenzkategorien, politischer Überzeugungen oder Generationen in den USA auftun. Im Moment der Abkapselung wird die Innenwelt zur Außenwelt, wird der Kapitän zum Präsidenten, der erste Offizier zur Oppositionspartei, das Boot zum »Staatsschiff« (Wolf 2013: 187) in seiner ganzen Komplexität. Der alte, explorative U-Boot-Film taucht dabei als eine mögliche Weltinterpretation wieder auf: In Crimson Tide spielen die Offiziere ein Spiel, in dem sie die Titel alter Hollywoodfilme dieses Genres raten müssen, wobei die Werke jeweils stellvertretend für die Weltsicht der Figuren stehen. Durch den systemischen Einschluss wird auf ästhetischer Ebene Erinnerbarkeit hergestellt: Das U-Boot wird zur Szenographie und somit zu einem konkreten Ort, der sich ins kollektive Imaginäre einzuschreiben vermag. Mit dem Begriff der Szenographie ist dabei ein kulturel- 
les Arsenal wiedererkennbarer und daher unmittelbar verständlicher, wenn auch in ihrer Bedeutung variierbarer und stets kontextbezogener Situationen bezeichnet, die eine Reihe von Erwartungen und Annahmen über mögliche Handlungen auslösen. Der von Umberto Eco geprägte Begriff geht auf Marvin Minskys Konzept des kognitiven Frames zurück (vgl. Minsky 1975), der in der aktuellen Forschung eine narratologische Wende erfahren hat: Durch Szenographien erscheint die Wirklichkeit als »in dramatische Episoden, in Mit- und Gegen-Spieler, in Handlungsmotive und -ziele, in antagonistische oder kointentionale Perspektiven und synreferentielle Bezirke aufgegliedert.« (Bauer 2003: 94) Da der Ozean selbst keinen konkreten Ort hat, sondern - jedenfalls abseits von Inseln und Küsten - in erster Linie als ungekerbter Raum erscheint, ist das Gedächtnis des Meeres auf solche Szenographien angewiesen: Es kann sich nicht an klassischen Erinnerungsorten orientieren, sondern muss über den Umweg der Narrativierung Räume etablieren, die dem Gedächtnis zugänglich sind. Die Szenographie des Atom-U-Boots entsteht eben in jenem Moment, in dem die Innenwelt des Bootes zu einem Spiegel der Außenwelt wird, zu der selbst gleichzeitig jeder Kontakt abgebrochen wurde. Kraft dieser Schließung verbinden sich die mentale Repräsentation des Kalten Kriegs in seiner Gesamtheit und der konkrete Handlungsraum des Films zu einer gebrochenen Allegorie, die den Kalten Krieg selbst als epistemologisches Problem kenntlich macht (vgl. Jameson 1992): Erinnerbar wird in erster Linie die Unsicherheit, in welcher ein in Latenz gehaltener Krieg seine potenziellen Teilnehmer zwingt.

Die Hermetik des U-Boots erklärt dabei retrospektiv auch die Fremdheit, die zwischen den beiden Supermächten im Kalten Krieg herrschte. In Bigelows K-19: The Widowmaker etwa wird die Mannschaft eines bereits so gut wie havarierten russischen Atom-U-Boots just in jenem Moment zum heroischen Kollektiv, in dem sie gemeinsam die Rettung durch die Amerikaner verweigert - allerdings nicht aus borniertem Nationalstolz, sondern in dem Wissen, dass die drohende Explosion des Bootes das fremde Schiff zerstören und so einen Weltkrieg auslösen könnte, also durch die imaginative Integration der Außenperspektive in die Innenwelt. Die Annäherung bringt den Tod, nur die Abkapselung rettet die Welt, auch wenn sie ein Selbstopfer bedeutet. Der Film stellt dabei insgesamt das Thema der politischen Sichtbarkeit ins Zentrum, besteht doch die Mission des russischen U-Boots darin, sich der Überwachung durch feindliche U-Boote zu entziehen, um in der Arktis eine Test-Rakete abzufeuern, die den Amerikanern die nukleare Kampfkraft der UdSSR vor Augen führt (zu den historischen Hintergründen vgl. Huchthausen 2002). Nach dieser gelungenen Inszenierung einer aus der Unsichtbarkeit hervorbrechenden Gewalt havariert das Schiff jedoch aufgrund eines defekten Kernreaktors und wird von einem amerikanischen Militärschiff entdeckt. Der finale Tauchgang wird zu einer bewussten Wahl des Vergessens, die Heldentat erweist sich als Selbstopfer, das nicht nur das eigene Leben, sondern auch die Möglichkeit des Gedenkens betrifft. Dieses wird, wie die Coda des Films zeigt, erst mit der Perestroika wieder möglich: Die Überlebenden des Unglücks treffen sich im Jahr 1989, nach dem Ende des Kalten Kriegs, vor dem Grab ihrer verstorbenen Kameraden, wobei das historische Datum das Ereignis 
als für Ost und West gleichermaßen relevant markiert. Erinnert wird auf diese Weise ein Heldenmut, der in diesem authentischen Fall gerade darin bestand, die Unsichtbarkeit des submarinen Raums zumindest auf absehbare Zeit als politische Notwendigkeit anzuerkennen. In dieser heldenhaften Selbstauslöschung besteht die erinnerungspolitische Paradoxie des Kalten Kriegs.

\section{Alte Boote, neuer Krieg}

Spätestens im Zuge der ersten Eskalationen im anhaltenden Konflikt zwischen Russland und der Ukraine hat in Presse und Politik die Denkfigur eines »neue[n] Kalte[n] Krieg[s]« (Hellmann 2015) an Popularität gewonnen. Auf diesen reagiert inzwischen auch der Hollywoodfilm, indem er die Erfolgskonzepte früherer Jahre wieder aufnimmt und etwa in Hunter Killer (USA 2018, R.: Donovan Marsh) eine Vielzahl der aus populären Filmen des Genres bekannten Motive und Handlungselemente zu einem neuen Narrativ verbindet, in dem dieses Mal ein US-amerikanisches U-Boot den russischen Präsidenten rettet. Während hier eine Aktualisierung der Vergangenheit in Form eines Reenactments des Kalten Kriegs stattzufinden scheint, das gegenwärtige Gefahren bannen soll, widmet sich die internationale, von der Kursk-Katastrophe inspirierte Koproduktion Black Sea (UK/USA/RU 2014, R.: Kevin Macdonald) einer Analyse des kulturellen Gedächtnisraums selbst, wobei die Wasserschichten der Tiefsee mit unterschiedlichen Kapiteln der jüngeren Geschichte assoziiert werden. Bemerkenswert erscheint der Film dabei auch deshalb, weil er das U-Boot von Beginn an als interkulturellen Raum gestaltet, in dem nun allerdings alte Konflikte erneut ausbrechen. Das Nachwirken der Vergangenheit wird auf diese Weise ebenso zum Thema wie die Funktionalisierung der Erinnerung im Rahmen gegenwärtiger Diskurse.

Der Film erzählt von dem U-Boot-Kapitän und Ex-Navy-Kommandanten Robinson (Jude Law), der am Anfang des Films von der Bergungsgesellschaft, für die er 11 Jahre gearbeitet hat, entlassen wird. Am Beginn steht damit metaphorisch das Ende jener Phase der Erinnerung, die auf den Kalten Krieg folgte. Von seinem ehemaligen Arbeitgeber wird Robinson mit nicht einmal 10.000 Pfund entschädigt und ist entsprechend frustriert. In einer Kneipe erfährt er jedoch von einem im Schwarzen Meer versunkenen deutschen U-Boot aus dem Zweiten Weltkrieg, das Gold an Bord gehabt haben soll - eine Zahlung des Deutschen Reichs an Stalin, die niemals ihr Ziel erreicht hat. Unterstützt von einem dubiosen Konzern, bemannt Robinson ein nahezu schrottreifes russisches Diesel-U-Boot aus den Zeiten des Kalten Kriegs mit einer halb englischen, halb russischen Besatzung, um den Schatz zu bergen. Der Film nimmt damit die Figur des Kapitäns als Abenteurer wieder auf, der hier nun jedoch die vergessene Geschichte der U-Boote selbst erforscht.

In den Tiefen des neuen globalpolitischen Konfliktgebiets des Schwarzen Meeres wird zwar das Gold gefunden, eskalieren aber auch die Spannungen zwischen den Kulturen. Die beiden Teile der Mannschaft, die durch eine Sprachbar- 
riere getrennt sind, versuchen sich gegenseitig umzubringen, um ihre Anteile am Schatz zu erhöhen, bis schließlich die eine Hälfte des auf dem Meeresgrund festliegenden Bootes von Russen, die andere von Engländern kontrolliert wird. Beide Parteien haben sich voneinander isoliert und ihre jeweiligen Territorien abgeriegelt; in der Heterotopie des U-Boots reproduziert sich so die anachronistische Frontstellung des Kalten Kriegs (Abb. 5). Am Ende des Films stellt sich heraus, dass der dubiose Geldgeber, der Robinson die Mission ermöglicht hat, der ganzen Mannschaft eine Falle gestellt hat: Nach dem Auftauchen sollen die Männer vom georgischen Küstenschutz festgenommen werden; das transnationale Unternehmen erhält das gesamte Gold. Das Schüren des Konfliktes zwischen den Nationen erscheint so als Taktik des Kapitals, wie insgesamt kapitalistische Interessen im Kern der historischen Dynamiken liegen. Der submarine Raum wird zugleich zu einer Zone kalkuliert hergestellter Unsichtbarkeit, in der die Verlierer der Gesellschaft, nachdem sie für die Konzerne die Drecksarbeit erledigt haben, zu Kriminellen umdefiniert werden.

Abbildung 5: In Kevin Macdonalds Black Sea reproduzieren russische und englische Zivilisten in einem schrottreifen U-Boot unter Wasser die Frontstellung des Kalten Kriegs.

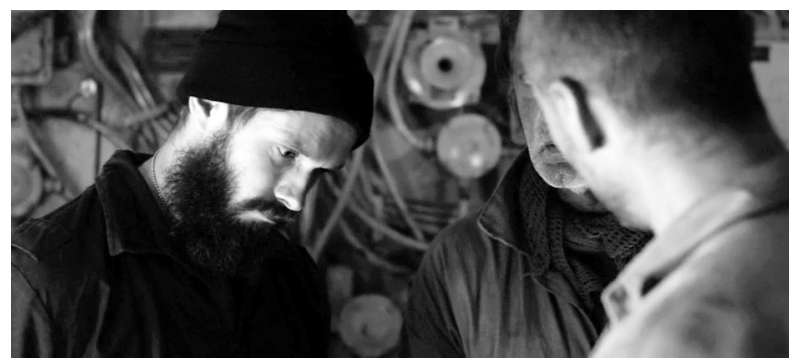

Das wenig subtile kapitalismuskritische Opfernarrativ, das die Figuren des Films immer wieder auch selbst artikulieren, erhält eine weitere Dimension dadurch, dass auch die Erinnerung selbst in Black Sea als ausbeutbar erscheint. In Form des rostigen russischen U-Boots wird die Vergangenheit hier allein aus dem Zweck reaktiviert, Gewinne zu generieren. Die Mission führt dabei durch unterschiedliche Zeitebenen, deren jeweilige Formen von Gewalt sich akkumuliert zu haben scheinen: Die Plünderungen des NS-Staates ziehen die gegenseitigen Aggressionen der Siegermächte nach sich, die wiederum von der Ausbeutung durch die international agierenden Konzerne der Gegenwart übertölpelt werden. Während Filme wie Kathryn Bigelows K-19: The Widowmaker den Kalten Krieg erinnerbar zu machen versuchen und Donovan Marsh in Hunter Killer seine Wiederkehr beschwört, macht Macdonald auf diese Weise die zerstörerischen Dimensionen dieser Zeit und die unproduktive Form deutlich, in der sie heute weiterwirkt. Das Personal seines Films reproduziert reflexhaft eine Semiotik der Feindschaft, die längst als obsolet erscheint, und verkennt auf diese Weise, dass sie mit den Ausgebeuteten anderer Länder regelrecht im gleichen Boot sitzt. Zu- 
gleich wird der > Wert $<$ jener Vergangenheit, die hier nautisch erschlossen wird, über die Metapher des Nazi-Goldes als vergiftet dargestellt. Erinnerung erweist sich auf diese Weise als bloßes Instrument in einem neuen, paranationalen und »post-memorialen« (vgl. Pence 2003) Machtdispositiv, als falsches, da letztlich destruktives Identitätsversprechen in einem ökonomischen Kalten Krieg, der auf radikaler Vereinzelung basiert - denn an Bord von Robinsons Schiff kämpfen in Wirklichkeit alle gegen alle. Von diesem neuen Kalten Krieg gilt auf andere, radikalere Weise, was Ramius in Red October schon vom alten Kalten Krieg sagte: »It's a war with no monuments, only casualties.«

\section{LITERATUR}

Bauer, Matthias (2003): Szenen gemeinsamer Aufmerksamkeit. Medien als Kulturpoetik. Zum Verhältnis von Kulturanthropologie, Semiotik und Medienphilosophie. In: Christoph Ernst/Petra Gropp/Karl Anton Sprengard (Hg.): Perspektiven interdisziplinärer Medienphilosophie. Bielefeld, S. 94-118.

Bordwell, David (1992): Cognition and Comprehension. Viewing and Forgetting in Mildred Pierce. In: Journal of Dramatic Theory and Criticism 6, H. 2, S. 183-197.

Brinckmann, Christine N./Lowry, Stephen/Wulff, Hans J. (2014): Von Zyklen, Reihen, Serien. Kleine Genres und ihre Verwandten. In: Montage AV 23, H. 1, S. 4-10.

Clancy, Tom (1984): The Hunt for Red October. Roman. Annapolis.

Eco, Umberto (1987): Lector in fabula. Die Mitarbeit der Interpretation in erzählenden Texten. München.

Engell, Lorenz (1989): Der gedachte Krieg. Wissen und Welt der Globalstrategie. München.

Erll, Astrid/ Wodianka, Stephanie (2008): Einleitung. Phänomenologie und Methodologie des 'Erinnerungsfilmsı. In: Dies. (Hg.): Film und kulturelle Erinnerung. Plurimediale Konstellationen. Berlin, S. 1-20.

Foucault, Michel (2005): Die Heterotopien/Der utopische Körper. Zwei Radiovorträge. Aus dem Franz. v. Michael Bischoff. Mit einem Nachwort v. Daniel Defert. Frankfurt a.M.

Gradinari, Irina (2018): Sedimentationen des Staates. Zu Ikonografie und Ideologie des Terrors von Die Hard bis Homeland. In: Dies. / Nikolas Immer / Johannes Pause (Hg.): Medialisierungen der Macht. Filmische Inszenierungen politischer Praxis. Paderborn, S. 331-354.

Grainge, Paul (2003): Introduction. In: Ders. (Hg.): Memory and Popular Film. Manchester/ New York, S. 1-20.

Grob, Norbert (2010): Kammern unter Wasser. Sieben Anmerkungen zum U-Bootfilm. In: Roman Mauer (Hg.): Das Meer im Film. Grenze, Spiegel, Übergang. München, S. 223-237.

Große Kracht, Klaus (2014): Zwischen Gedächtnis und Geschichte. Erinnerungsorte als "Heterotopien der Zeitu. In: Stefan Berger/Joana Seiffert (Hg.): Erinnerungsorte. Chancen, Grenzen und Perspektiven eines Erfolgskonzeptes in den Kulturwissenschaften. Essen, S. 57-72. 
Hellmann, Günther (2015): Ein neuer Kalter Krieg? Russland, die NATO und der Regionalkonflikt in der Ukraine. In: Reader Sicherheitspolitik 3; online unter: https:// www.fb03.uni-frankfurt.de/76345838/Artikel_Ein_neuer_Kalter_Krieg.pdf? [Stand: 1.10.2020].

Horn, Eva (2007): Den Krieg als Spiel denken. Boyscouts und Wargames. In: Claus Pias/Christian Holtorf (Hg.): Escape! Computerspiele als Kulturtechnik. Dresden, S. 215-224.

Dies. (2009): Der Anfang vom Ende. Worst-Case-Szenarien und die Aporien der Voraussicht. In: Archiv für Mediengeschichte 9: Gefahrensinn, S. 91-100.

Huchthausen, Peter (2002): K-19 und die Geschichte der russischen Atom-U-Boote. Hamburg.

Jameson, Fredric (1992): The Geopolitical Aesthetic. Cinema and Space in the World System. Bloomington.

Koldau, Linda Maria (2010): Mythos U-Boot. Stuttgart.

Dies. (2012): Submarine Films as Aesthetic Reflection of War History and War Strategy. In: Søren Fauth/Kasper Green Krejberg/Jan Süselbeck (Hg.): Repräsentationen des Krieges. Emotionalisierungsstrategien in der Literatur und in den audiovisuelIen Medien vom 18. bis zum 21. Jahrhundert. Göttingen, S. 102-118.

Latour, Bruno (1986): Visualization and Cognition. Drawing Things Together. In: Knowledge and Society: Studies in Sociology of Culture Past and Present 6, S. 1-40.

Mann, Martin (2018): Die Interkulturalität des Krieges. In: Eva Wiegmann (Hg.): Diachrone Interkulturalität. Heidelberg, S. 339-350.

Minsky, Marvin (1975): A Framework for Representing Knowledge. In: Patrick Henry Winston (Hg.): The Psychology of Computer Vision. New York.

Nanz, Tobias (2016): Zimmer mit kolossaler Aussicht. War Rooms als imaginäre Aushandlungsorte von Störungen. In: Behemoth. A Journal on Civilisation 9, H. 1: Imaginationen der Störung, S. 38-57.

Pence, Jeffrey (2003): Postcinema/Postmemory. In: Paul Grainge (Hg.): Memory and Popular Film. Manchester/ New York, S. 237-256.

Rayner, Jonathan (2007): The Naval War Film. Genre, History and National Cinema. Manchester/ New York.

Ritzer, Ivo (2010): Heterotopisches, Abjektes, Apokalyptisches. Submarine Welten im Film. In: Roman Mauer (Hg.): Das Meer im Film. Grenze, Spiegel, Übergang. München, S. 268-280.

Robin, Ron (2001): The Making of the Cold War Enemy. Culture and Politics in the Military- Intellectual Complex. Princeton.

Seed, David (1999): American Science Fiction and the Cold War. Literature and Film. Edinburgh.

Sharp, Joanne (1998): Reel Geographies of the New World Order. Patriotism, Masculinity, and Geopolitics in Post-Cold War American Movies. In: Gearóid Ó Tuathail/ Simon Dalby (Hg.): Rethinking Geopolitics. New York/London, S. 152-169.

Vehlken, Sebastian (2018): Finding the Boomers. Der Anti-U-Boot-Krieg und die Operationalisierung der Ozeane im Kalten Krieg. In: Lars Nowak (Hg.): Medien - Krieg Raum. Paderborn, S. 357-376.

Wolf, Burkhardt (2013): Fortuna di mare. Literatur und Seefahrt. Zürich/Berlin. 